\title{
The Gardner correlation length scale in glasses
}

\author{
M J Godfrey ${ }^{1}$ and M A Moore ${ }^{1}$ \\ ${ }^{1}$ Department of Physics and Astronomy, University of Manchester, Manchester M13 \\ 9PL, United Kingdom
}

\begin{abstract}
The Gardner length scale $\xi$ is the correlation length in the vicinity of the Gardner transition, which is a transition in glasses where the phase space of the glassy phase fractures into smaller sub-basins on experimental time scales. We argue that $\xi$ grows like $\sqrt{B_{\infty} / G_{\infty}}$, where $B_{\infty}$ is the bulk modulus and $G_{\infty}$ is the shear modulus, both measured in the high-frequency limit of the glassy state. We suggest that $\xi$ might be inferred from stress-stress correlation functions, which is more practical for experimental investigation than studying two copies of the system, which can only be done in numerical simulations. Our arguments are illustrated by explicit calculations for a system of disks moving in a narrow channel, which is solved exactly by transfer matrix techniques.
\end{abstract}

Keywords: colloidal glasses, classical phase transitions, structural correlations 


\section{Introduction}

The Gardner transition [1] is a transition to a state of full replica symmetry breaking (FRSB) from a state with one-step replica symmetry breaking: it exists in exotic spin glass models such as the Potts spin glass [2] or the $p$-spin glass model [1], at least when studied in the mean-field approximation. The recent revival of interest in it is because structural glasses seem to have within their glassy state features similar to the loss of ergodicity expected at the Gardner transition; for a review, see [3]. To date, most of the numerical studies of it have focused on finding similarities between the features observed in dimensions $d=2$ or $d=3$ and those predicted by the mean-field theory of replica symmetry breaking, which is certain to be valid only in infinite dimensions [4, 5, 6, 7]. This has involved looking at the overlaps of the states in copies $A$ and $B$ of the glassy system, which at some initial time have the particles in the same positions, but with different initial velocities. While this device is useful in numerical simulations for detecting the onset of non-ergodicity, it is not easy to mimic in a real experiment.

The Gardner transition in finite dimensions is in the universality class of the Almeida-Thouless transition of spin glassses [8]. According to some [9, 10, 11], the Almeida-Thouless transition is at best an "avoided" transition for all dimensions $d \leq 6$. If so, the Gardner transition will also be an "avoided" transition for dimensions $d \leq 6$, in that the Gardner correlation length can only grow large for hard or soft spheres or disks [4, 12, 13, 7] and there will be no actual divergence of this length scale as at a real transition or as found in the mean-field limit, which is exact only as $d \rightarrow \infty$.

In this paper, we shall obtain a simple expression for the Gardner length scale which explains in a quantitative way the circumstances in which it can become large. Furthermore, we shall show that the length scale can be obtained by experiments on shear-shear or stress-stress correlations, and does not require the study of two copies of the system, $A$ and $B$. Finally we show that at densities above that of the Gardner transition there are structural changes in the system that are not described by the mean-field calculations.

In the "state-following" Gardner transition in structural glasses one studies the transition within a given glass state, that is, on time scales short compared to the alpha relaxation time so that the atoms will not wander far from their initial positions. This restriction on the motion of the atoms allows us to derive an effective elastic model for the glass. We find that the Gardner length scale $\xi$ varies approximately as $\sqrt{B_{\infty} / G_{\infty}}$, where the bulk compressibility $B_{\infty}$ and the shear modulus $G_{\infty}$ are the values these elastic moduli take at high frequencies or on time scales less than the alpha relaxation times. If the ratio of the moduli becomes large, the length scale $\xi$ becomes large. This ratio does indeed become large at the $J$-point of the glass [14, 15] and so $\xi$ is large if the state is "close" to the $J$-point [16, 17, 12, 13]; otherwise it will be small. We shall illustrate our results by explicit calculations for a system of disks moving in a narrow channel, whose thermodynamic properties can be obtained via the transfer matrix technique. We believe that our results are generic and give a simple argument that suggests that they 
should extend to both two and three dimensional glasses.

In Sec. 2 we describe the simple model system of disks in a narrow channel which we use to motivate our arguments. In Sec. 3 we briefly discuss how our results vary with the width of the channel (but only over a restricted range). The determination of the Gardner length scale from the strain-strain correlation length is given in Sec. 4 and we derive a simple effective Hamiltonian which describes its behavior. Finally in Sec. 5 we discuss the implication of our results for hard and soft disks in higher dimensions.

\section{The model: disks in a narrow channel}

The system of disks in a narrow channel has previously been studied by ourselves and others in some detail [18, 19, 20, 21, 22, 23, 24, 25, 26, 27, 28, 29, 30]. Being effectively one dimensional, it does not have any true phase transitions. It has many typical glass features, such as relaxation times that grow rapidly with increasing packing fraction, [18, 20], the remnants of Kauzmann behavior [19], and an avoided Gardner transition [29]. Examples of some configurations of the disks are given in Fig. 1. The channel width available to the centers of the disks is defined as $h=H_{d}-\sigma$, where $\sigma$ is the diameter of a disk and $H_{d}$ is the width of the channel; we parametrize $h$ by $h=\sqrt{3} \sigma / 2+\epsilon \sigma$, where $0<\epsilon<(1-\sqrt{3} / 2)$, so that the disks cannot pass each other. The packing fraction $\phi$ is defined as $\phi=N \pi \sigma^{2} /\left(4 H_{d} L\right)$, where $N$ is the number of disks in a channel of length $L$. The center of the $n$th disk has co-ordinates $\left(x_{n}, y_{n}\right)$, where $y_{n}$ is measured from a line down the center of the channel. Thus a disk which touches a channel wall will have $y_{n}= \pm h / 2$. The transfer matrix has been used to obtain numerically exact values for the thermodynamic properties and correlation functions of the model [19]. The Gardner correlation length $\xi$ has already been determined from the eigenvalues of the transfer matrix [19, 29] for one value of the channel width, $h=0.95 \sigma$. It peaks at a value of $\xi \approx 30$ at a packing fraction $\phi_{G} \approx 0.8049$ for $h=0.95 \sigma$ (see Fig. 2). However, by decreasing the channel width $(\epsilon \rightarrow 0)$ we can make the Gardner length scale $\xi$ as large as we wish at the avoided transition, so that we can adjust the extent to which the transition is avoided, as demonstrated in Sec. 3. In the simulations in two and three dimensions it has proved to be difficult to determine the Gardner length scale for hard disks or spheres [7].

The dynamics in this system start to slow as "zigzag" order sets in above a packing fraction $\phi=\phi_{d} \approx 0.48$ [20, 19, 18] for $h=0.95 \sigma$, which is the width mostly used in this paper except in Sec. 3. Zigzag order is characterized by successive values of $y_{i}$ taking opposite signs and is a form of bond orientational order. The zigzag order can be interrupted by defects where successive $y_{i}$ are of the same sign; the correlation length $\xi_{2}$ for zigzag order is approximately half the average distance between these defects [20]. These defects play an important role in the dynamics of the system. The spacing between these defects increases rapidly with increasing packing fraction $\phi$, such that for $h=0.95 \sigma, \xi_{2}$ passes 2000 at $\phi=0.7206$ and reaches $\xi_{2}=2.3 \times 10^{6}$ at $\phi=0.76$. In this study we focus on even higher packing fractions so that our systems have essentially 

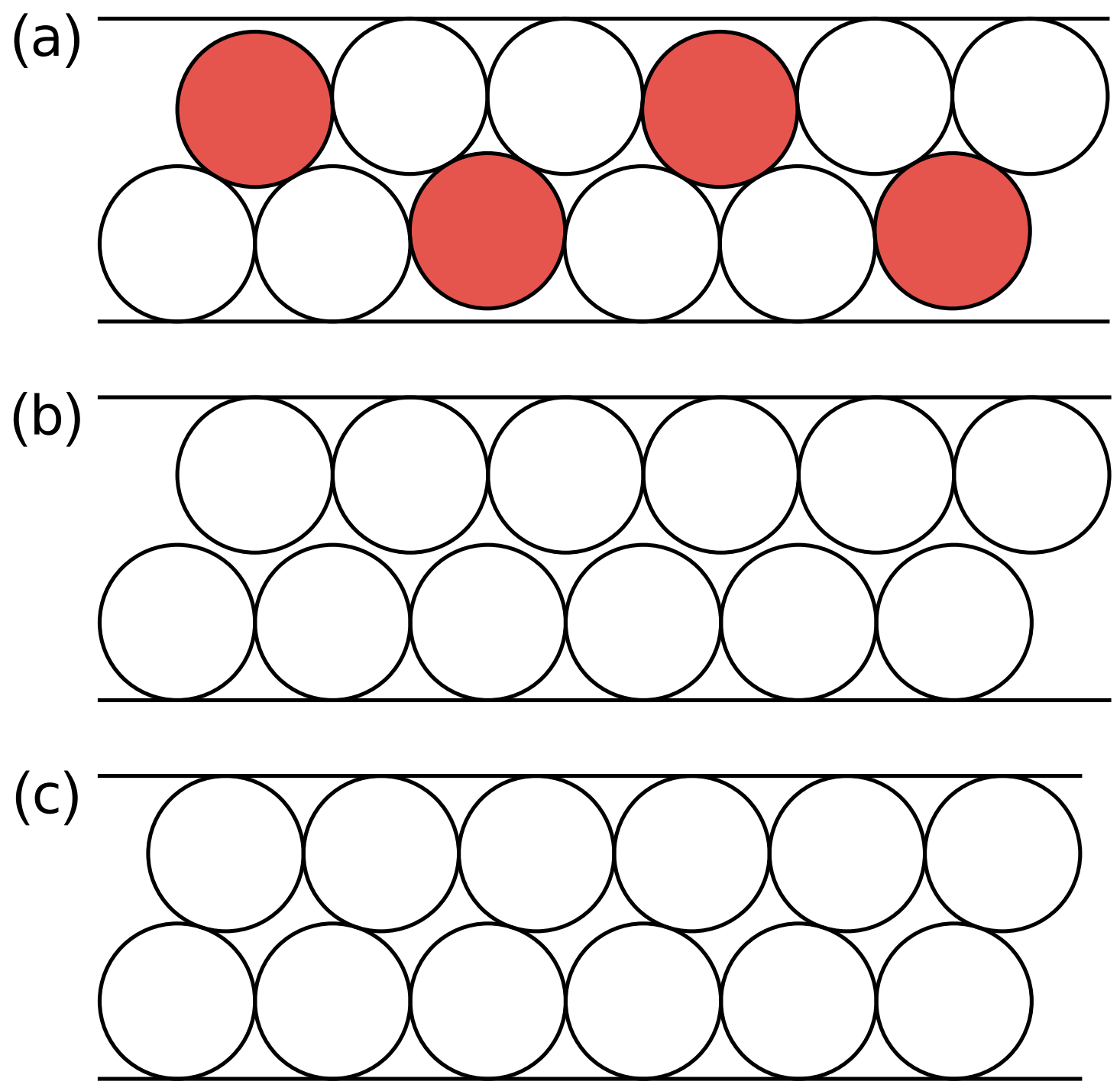

Figure 1. (a) Configuration of disks at the maximum possible packing fraction $\phi_{\max } \simeq 0.8074$ when $h=0.95 \sigma$. The red disks do not touch the sides of the channel. (b) Configuration of the disks at a density $\phi_{K} \simeq 0.8055$. This is the highest density state which can be reached when all the disks touch a channel wall. Note that the disks in the upper row can be translated with respect to those in the lower row, as shown in (c).

perfect zigzag order.

We used the transfer matrix to calculate the equation of state of the system [19]. This is the relation between the force $F$ which has to be applied to pistons at both ends of the system to confine the $N$ particles within it so that the system's length $L$ corresponds to a packing fraction $\phi$. Fig. 2 shows the equation of state for the case $h=0.95 \sigma$. The force $F$ appears to diverge at the packing fraction $\phi_{K}=\pi \sigma /\left(2 H_{d}\right)$, which is the packing fraction of the configurations in Fig. 1(b) or Fig. 1(c). The equation of state for the 


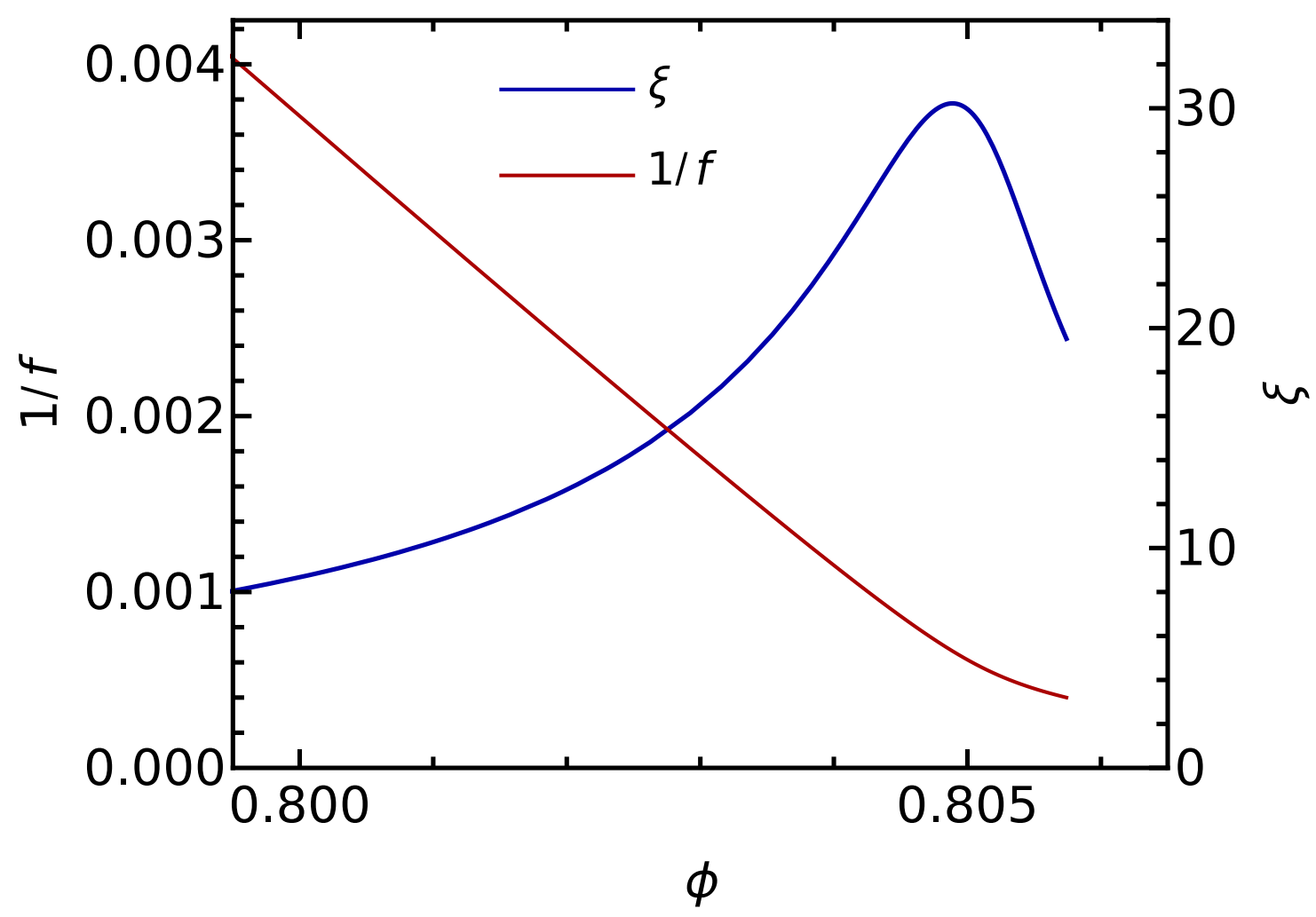

Figure 2. Red: Plot of $f^{-1}$ where $f \equiv \beta F \sigma$ against packing fraction $\phi$ for disks in a channel of width $h=0.95 \sigma$. Blue: On the right axis we have plotted $\xi$. It peaks at the packing fraction $\phi_{G}(\approx 0.8049)$, which is where the equation of state curve starts to deviate significantly from a straight line.

dimensionless force $f \equiv \beta F \sigma$ is approximated fairly well by $1 / f=\left(L-L_{K}\right) / N$, where $L_{K}=N \sigma / 2$, but the divergence in $f$ predicted by this approximation at $\phi=\phi_{K}$ is avoided, as the curve veers off around the density $\phi_{G}$ : $f$ truly diverges only at the maximum possible density $\phi_{\max }$, which corresponds to the crystalline arrangement shown in Fig. 1(a). The equation of state changes its form just above $\phi_{G}$, when some disks become locked into positions away from the channel wall (as in Fig. 1(a)). We use the notation $\phi_{K}$ as the correlation length for the zigzag order $\left(\xi_{2}\right)$ appears to diverge as $\phi \rightarrow \phi_{K}$ : it grows approximately as $\sim \exp ($ const. $\times f)$ [19]. The departure of the equation of state from the straight line at the Gardner packing fraction $\phi_{G}$ indicates that for $\phi>\phi_{G}$ the glass system has acquired new structural features. In Fig. 3 we have plotted the average density $\rho_{1}(z)$ as a function of the distance from the channel wall to show the emergence of one of these new structural features when $\phi>\phi_{G}$ : an increased probability of disks being found at distance $\approx \epsilon \sigma$ away from the wall.

In Fig. 2 we show how the correlation length $\xi$ obtained from the logarithm of the ratio of the third eigenvalue of the transfer matrix to the first eigenvalue varies with the 


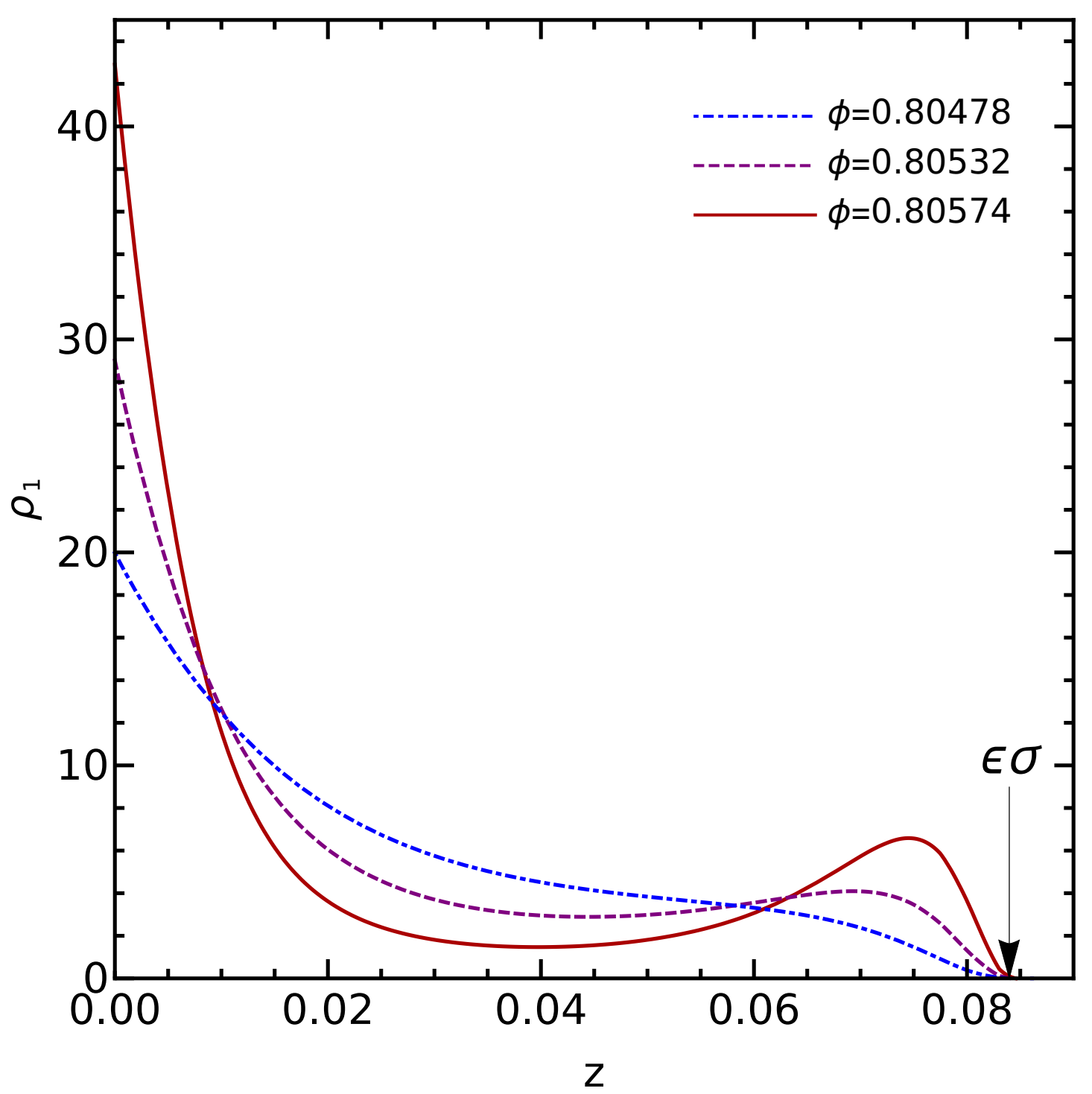

Figure 3. Plot of the density $\rho_{1}(z)$, where $z=h / 2-y$ is the distance of a disk from the wall for three different values of the packing fraction $\phi$ when the channel width $h=0.95 \sigma$. As the packing fraction increases past $\phi_{G} \approx 0.8049$, there is an increasing probability to find disks away from the wall. An arrow marks $z=\epsilon \sigma$, which corresponds to the positions of the shaded disks in Fig. 1(a).

packing fraction $\phi$. (The similar expression involving the second eigenvalue determines the length scale $\xi_{2}$ of zigzag order.) The most striking feature of the behavior of $\xi$ as a function of $\phi$ is the peak. The peak occurs at the packing fraction $\phi_{G}$ where some of the disks start to be locked into positions at distances $\epsilon \sigma$ away from the channel walls, as in the state of maximum density in Fig. 11(a) [19]. A correlation length which rises to a maximum value and then falls is typical of an avoided transition. In Ref. [29] we identified the peak in $\xi$ with the "avoided" Gardner transition and showed that the 


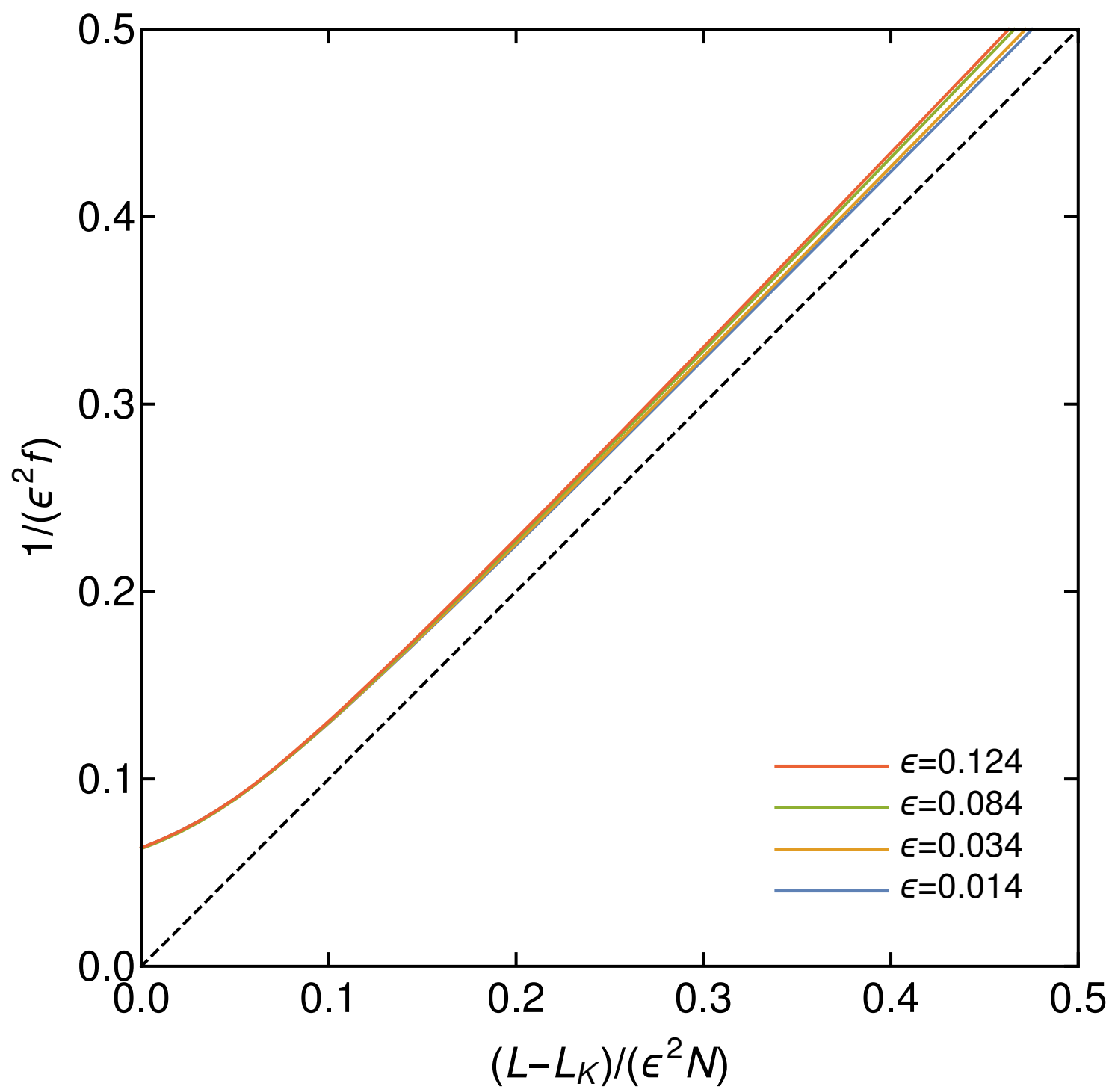

Figure 4. Plot of $1 /\left(\epsilon^{2} f\right)$ versus $\left(L-L_{K}\right) /\left(\epsilon^{2} N \sigma\right)$, where $f \equiv \beta F \sigma$ and $L_{K}=N \sigma / 2$ is the length of the system in the configurations shown in Fig. 1(b) or Fig. 1(c). The equation of state continues to negative values of $\left(L-L_{K}\right) /\left(\epsilon^{2} N\right)$ and reaches $-1 / 6$ when $1 /\left(\epsilon^{2} f\right)$ becomes 0 , which corresponds to $\phi=\phi_{\max }$. (We have so little data for the region $L<L_{K}$ that we have not plotted any of it.) The dashed line corresponds to $1 / f=\left(L-L_{K}\right) / N$. The width of the channel is related to $\epsilon$ by $h=\sqrt{3} \sigma / 2+\epsilon \sigma$.

length scale associated with the Gardner transition defined via the overlap of two copies of the system $A$ and $B$ was indeed described by $\xi$. For $h=0.95 \sigma$, the peak occurs at a packing fraction $\phi_{G} \approx 0.8049$, which is lower than $\phi_{K} \approx 0.8055$. 


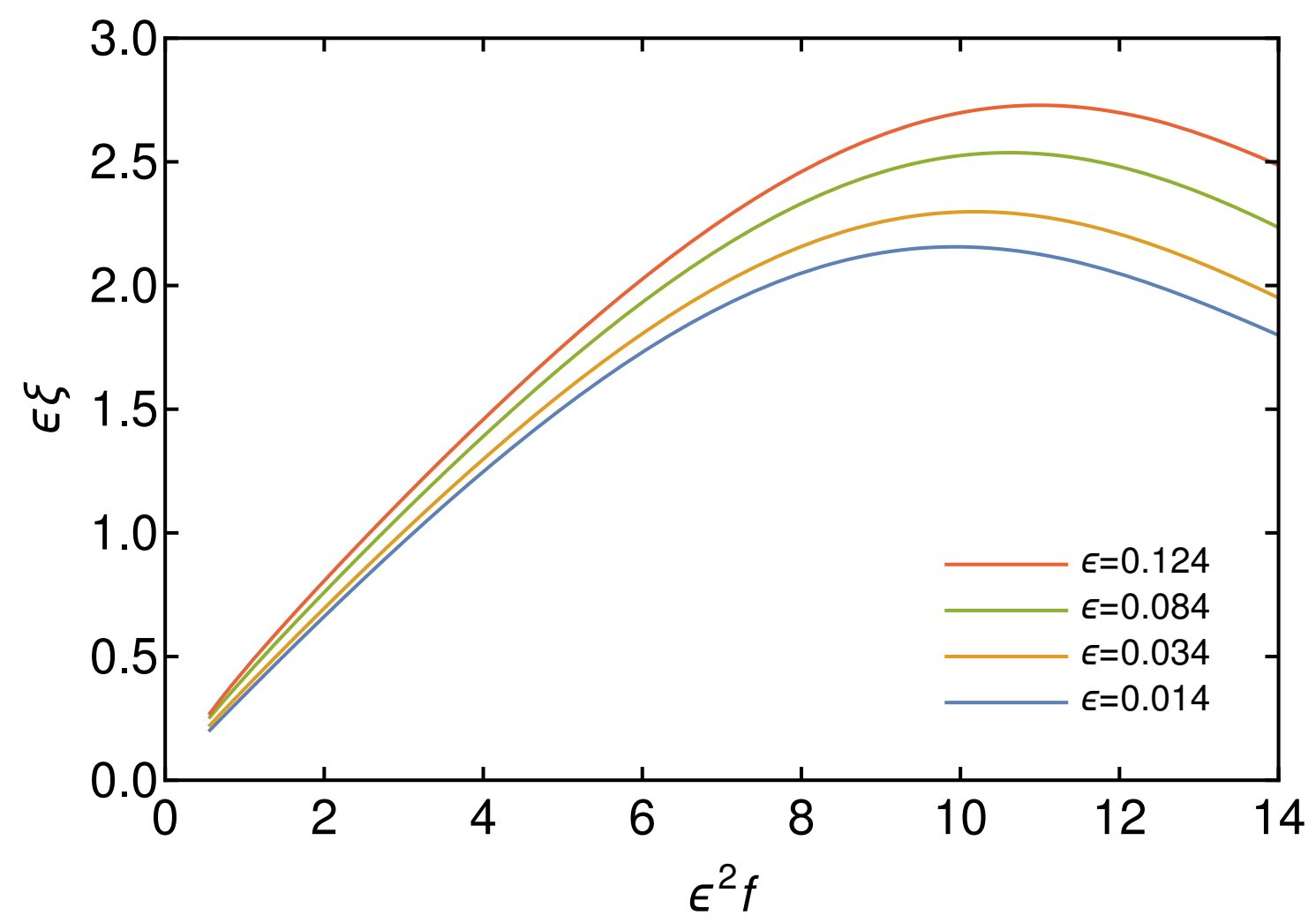

Figure 5. Plot of the scaled correlation length $\epsilon \xi$ versus the scaled force $\epsilon^{2} f$, for various values of $\epsilon$.

\section{Dependence on channel width}

In this section we shall give results for the behavior of the equation of state and the Gardner length $\xi$ for general values of the width parameter $\epsilon$, where $\epsilon=h / \sigma-\sqrt{3} / 2$. Fig. 4 shows results for the equation of state in the region $\phi<\phi_{K}$ for several values of $\epsilon$. The dashed line is a plot of the dimensionless force $f=\beta F \sigma$ as $1 / f=\left(L-L_{K}\right) / N$; Fig. 4 shows this is is accurate to order $\epsilon^{2}$. Notice that there are other shortcomings in this approximate equation of state. It fails when $\phi>\phi_{G}$ due to the changes in the structure of the glass above $\phi_{G}$.

In Fig. 5 we have studied $\xi$ as a function of $\epsilon$ and the dimensionless force $f$. If one plots $\epsilon \xi$ against $\epsilon^{2} f$, there seems to be a rather rough "collapse" of the data onto a universal curve. We suspect that it is only in the limit $\epsilon \rightarrow 0$ that the collapse becomes convincing. In that limit, the difference between $\phi_{K}$ and $\phi_{\max }$ decreases as $\epsilon^{2}$, and $\phi_{G}$ (the packing fraction where $\xi$ peaks as a function of $\phi$ ) gets closer to $\phi_{K}$. According to Fig. 5, the growth of $\xi$ as $\epsilon \rightarrow 0$ has the approximate scaling form $\epsilon \xi=X\left(\epsilon^{2} f\right)$, with $\xi \approx \epsilon f$ when $\epsilon^{2} f$ is small. In the region where $\xi$ is large and increasing with $f$ but $\epsilon^{2} f$ is still small, we find $\operatorname{Var} x_{N N} \sim \epsilon / f$ [19], $B_{\infty} \sim f^{2}, G_{\infty} \sim 1 / \epsilon^{2}$, and $\xi \rightarrow 0.5 \sqrt{B_{\infty} / G_{\infty}} \sim \epsilon f$. 
On taking $\epsilon \rightarrow 0$, the avoided transition become sharper, and more similar to a true transition. Exactly at the peak, $f \epsilon^{2}$ is of order unity, according to Fig. 5, so that $\xi_{G}$, the Gardner length scale at the peak, varies as $\sim 1 / \epsilon$, which shows that the Gardner length can be made arbitrarily large by choosing $\epsilon$ to be sufficiently small.

\section{The strain-strain correlation length}

We have studied the strain-strain correlation function $G_{x x}(s)$ defined by $G_{x x}(s) \equiv$ $\left\langle\tilde{x}_{i} \tilde{x}_{i+s}\right\rangle$. Here $\tilde{x}_{i} \equiv\left(x_{i+1}-x_{i}-\left\langle x_{i+1}-x_{i}\right\rangle\right)$ is the $x$-component of the strain between particle $i+1$ and particle $i$. Note that $\left\langle\tilde{x}_{i}\right\rangle=0$. The average spacing between the disks along the $x$ axis is $\left\langle x_{i+1}-x_{i}\right\rangle=L / N$. Fig. 6. shows that $G_{x x}(s)$ decays with $s$ on the Gardner length scale $\xi$ for large $s$ at any packing fraction $\phi$ and that for $\phi<\phi_{G}$ it is accurately described by the equation

$$
G_{x x}(s) \approx(-1)^{s} \operatorname{Var} x_{N N} \exp (-s / \xi)
$$

Var $x_{N N}$ denotes the variance of the nearest-neighbor spacing $x_{i+1}-x_{i}$ and equals $G_{x x}(0)$ [19]. The oscillation in sign arises from the fact that the two rows of disks tend to move independently of each other so that if one nearest-neigbor distance is increased, the following one will decrease. $\xi$ in Eq. (1) is equal to that calculated from the logarithm of the ratio of the third eigenvalue of the transfer matrix to the first eigenvalue of the transfer matrix.

Notice that studying the strain-strain correlation function can be done within a single copy of the system. When using the transfer matrix technique, it is simple to determine the strain-strain correlation function. However, when doing a molecular dynamics simulation in higher dimensions it might be easier to study the stress-stress correlation function. It seems natural to expect that both types of correlation function will decay exponentially on the same length scale $\xi$.

For understanding the behavior of the strain-strain correlation function at densities $\phi<\phi_{G}$, we shall find it useful to introduce an effective Hamiltonian. Our system has the feature that the disks cannot pass each other, so their ordering persists forever: they are "caged" even in the low density region. (In three dimensions caging is only effective on time scales less than the alpha relaxation time.) With the effective Hamiltonian

$$
\mathcal{H}=\frac{1}{2} B_{\infty} \sum_{n}\left(\tilde{x}_{n}+\tilde{x}_{n+1}\right)^{2}+\frac{1}{2} G_{\infty} \sum_{n}\left(\tilde{x}_{n}-\tilde{x}_{n+1}\right)^{2}
$$

Eq. (1) is recovered as an equality, provided we choose $B_{\infty}$ and $G_{\infty}$ so that

$$
\xi=\frac{1}{2 \operatorname{arctanh}\left(\sqrt{G_{\infty} / B_{\infty}}\right)}
$$

and $\operatorname{Var} x_{N N}=1 /\left(4 \beta \sqrt{B_{\infty} G_{\infty}}\right)$. The first term in brackets in Eq. (2) involves $\left(\tilde{x}_{n}+\tilde{x}_{n+1}\right) \equiv x_{n+2}-x_{n}-2 L / N$, while the second term involves $\left(\tilde{x}_{n}-\tilde{x}_{n+1}\right) \equiv$ 


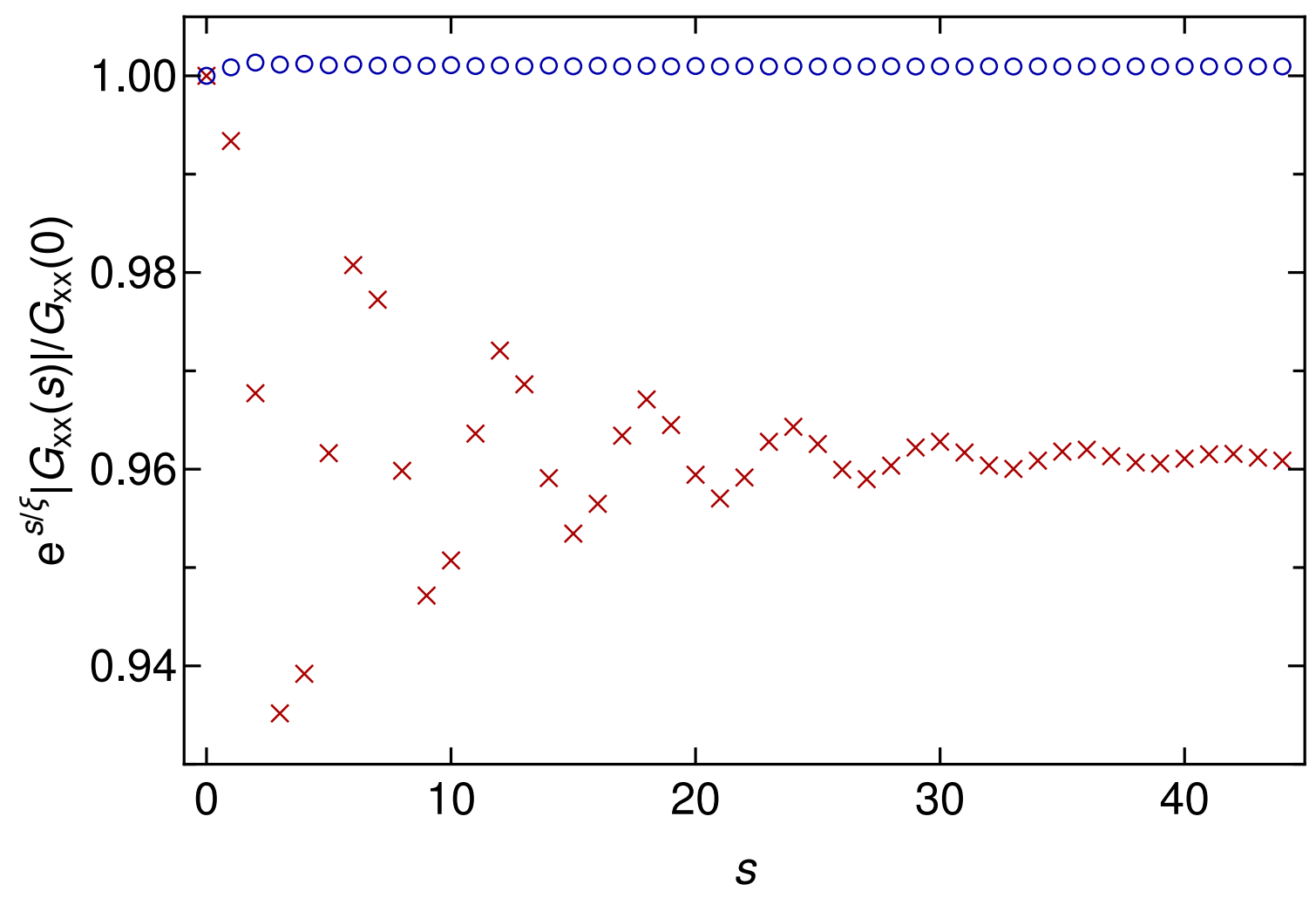

Figure 6. The ratio $\exp (s / \xi)\left|G_{x x}(s)\right| / G_{x x}(0)$ versus separation $s$ at two values of $f$; one below the peak in the $\xi$ versus $f$ plot (see Fig. 5 ) at $f=500$ (blue circles), where $\xi \approx 14.9$ and one above beyond the peak at $f=2500$ (red crosses), where $\xi \approx 19.5$ for the width $h=0.95 \sigma$. If Eq. (1) were a perfect fit, the ratio would be unity for all $s$.

$2 x_{n+1}-x_{n}-x_{n+2}$. Because of the zigzag order, the first term is the spacing between disks in the same row. This separation is actually on average smaller than that between disks $n$ and $n+1$ [19]. If the pistons are pushed in, this elastic term measures the free energy cost of compressing the system and the coefficient $B_{\infty}$ is the bulk modulus of the system. The second term represents the free-energy cost of a shear displacement in which the disks in the top row slide with respect to those in the bottom row, as shown in Fig. 1. This can be recognized from the fact that it is non-zero when $x_{n+1}$ which is in (say) the top row, moves away from the mid-point of the two disks $n$ and $n+2$ in the bottom row, $\left(x_{n}+x_{n+2}\right) / 2$, as in Fig. 1(b). Its coefficient will be the shear modulus $G_{\infty}$ of our system. The term represents an effective coupling, as the entropy of the system is greatest when the disks in one row hover above the dips between adjacent disks in the other row; thus, it is a many-body rather than just a pairwise effective interaction.

Equation (2) will have corrections to it that involve higher powers of $\tilde{x}_{n}$. At densities above $\phi_{G}$, Fig. 6 shows that $G_{x x}(s)$ has features for $s<\xi$ which are not captured in the simple exponential form of Eq. (1) predicted by Eq. (2), but this form remains accurate to $\approx 96 \%$ at the highest density studied, which corresponds to $f=2500$. The 
departures from the simple exponential decay are consequences of the structural changes that emerge for $\phi>\phi_{G}$.

Thus the effective Hamiltonian of Eq. (2) provides a good description of the system for $\phi<\phi_{G}$. We shall interpret it in two ways. The first is to introduce a new variable, the "tilt" $t_{i}=\tilde{x}_{i} / \operatorname{sign}\left(y_{i}\right)$. The $\operatorname{sign}\left(y_{i}\right)$ term removes the $(-1)^{s}$ sign oscillation in Eq. (1), so that $\left\langle t_{i} t_{i+s}\right\rangle \sim \exp (-s / \xi)$, a simple exponential decay. In terms of the variables $t_{n}$, the effective Hamiltonian is

$$
\mathcal{H}=\frac{1}{2} B_{\infty} \sum_{n}\left(t_{n+1}-t_{n}\right)^{2}+\frac{1}{2} G_{\infty} \sum_{n}\left(t_{n+1}+t_{n}\right)^{2} .
$$

Note that if one is working in the region where $B_{\infty} \gg G_{\infty}$, the first term is minimized by having the variables $t_{n}$ of the same sign. Furthermore, if the variation of $t_{n}$ with $n$ is small one can approximate $t_{n+1}-t_{n} \rightarrow \partial t(x) / \partial x$ and $t_{n+1}+t_{n} \rightarrow 2 t(x)$ so that the continuum version of Eq. (4) is

$$
\mathcal{H}=\int d x\left[\frac{1}{2} B_{\infty}(\partial t(x) / \partial x)^{2}+\frac{4}{2} G_{\infty} t(x)^{2}\right] .
$$

From Eq. (5) it is easy to see that there will be a correlation length $\xi \rightarrow 0.5 \sqrt{B_{\infty} / G_{\infty}}$.

The second way of understanding the origin of the length scale $\xi$ will be useful in higher dimensions. It is to Fourier transform the strain-strain correlation function of Eq. (1), assuming that the particle are on average spaced by $a=L / N$. The result for the Fourier transform $\tilde{G}_{x x}(k)$ for $\xi$ large is

$$
\tilde{G}_{x x}(k)=\frac{T}{4 G_{\infty}+2 B_{\infty}[1+\cos (k a)]} .
$$

For $k a=\pi+q a$ with $q a \ll 1$, the denominator is proportional to $\left(1 / \xi^{2}+(q a)^{2}\right)$. Thus the long length scale $\xi$ is associated with a softening of the elastic modes at the "zone boundary", $k=\pi / a$. It is the many-body term involving $G_{\infty}$ in Eq. (2) which stabilizes the system against the free sliding of one row with respect to the other row.

\section{Discussion}

We will now discuss how this argument might extend to glasses in dimensions $d=2$ and $d=3$. On time scales less than the alpha relaxation time the particles in the glass are caged. Effective Hamiltonians similar to that of Eq. (2) can be written down for the glass state in higher dimensions, as that effective Hamiltonian is just one of the many possible finite-difference forms that reduce to continuum elasticity theory in the long-wavelength limit. Furthermore, in the region near the J-point, the number of nearest-neighbors will be close to $2 d$ on average, rather as on a simple-cubic lattice for $d=3$ or a square lattice in $d=2$ [14, 31, 16, 17]. A simple-cubic or a square lattice with only pairwise interactions between nearest-neigbors on the lattice is unstable against sliding one plane (or row) of particles with respect to the rest. This kind of instability is at the wavevector $k=\pi / a$, 
where $a$ is the lattice spacing. It is the analogue of sliding the top row of disks with respect to the bottom row in the disks in the channel system. Indeed, as in our channel system, it is only the existence of next-nearest-neighbor interactions which makes such cubic systems stable and gives rise to a non-zero shear modulus $G$. In glasses the situation will be more complicated, as there will be no freely sliding planes of spheres; in fact, no well-defined planes of spheres. However, near the J-point a snapshot of the system would show that it is close to a state of marginal stability; $G$ is actually zero at the J-point itself. It seems natural to expect that at wave-vectors $|\mathbf{k}|$ that correspond to a displacement similar to that near $\pi / a$, there will be a peak in the strain-strain correlation function, as in Eq. (6) due to the expected softening at such a wavevector. In fact, such a peak may have already been observed in the stress-stress correlation functions studied in Ref. [32] for the case of a truncated Lennard-Jones potential. In that work, no peak was visible in states of a lower packing fraction $\phi=0.699$, but there was a striking peak for states with a packing fraction $\phi=0.80$, which is closer to the J-point of this system. The length scale $\xi$ can be extracted from the peak in the strainstrain correlation function (the higher dimensional analogue of Eq. (6)) and provided $B_{\infty} \gg G_{\infty}$ it should vary as $\sim \sqrt{B_{\infty} / G_{\infty}}$. Alas, simulations on hard or soft spheres have not yet been sufficiently developed to allow this relationship to be investigated for three dimensional systems [3].

It has not escaped our notice that the softening as the J-point is approached will contribute to the "boson peak" effect [33]: a boson peak arises when there are more low frequency phonon modes than might have been expected from just the long-wavelength elastic modes included in the Debye approximation.

For soft spheres the J-point [12, 13] has been shown to lie within the Gardner phase. We believe that the Gardner transition is an avoided transition in physical dimensions so we suspect that that Gardner-like features will only be seen at temperatures and packing fractions which are close to the J-point, (which is a $T=0$ point). Support for

this idea and also our formula $\xi \sim \sqrt{B_{\infty} / G_{\infty}}$ comes from the work of Refs. [34, 35]. There it was found that for packing fractions larger than $\phi_{J}$, there was a length scale in jammed states of soft spheres which has had the same dependence on the elastic moduli as in this paper. This length scale marked the crossover in the response to a force dipole between the fluctuation-dominated near field and the elastic-continuum far-field. This length is also the scattering length associated with the "anomalous modes" that are important near the J-point. Dynamical criticality at finite temperatures near the jamming transition has been extensively discussed in [36, 37].

\section{Acknowledgments}

We thank Ludovic Berthier, and Camille Scalliet for useful exchanges, Kamran Karini, Craig Maloney and Steve Teitel for information on the work in Ref. [32, and Eric De Giuli for pointing out the features mentioned in the previous paragraph. 
The Gardner correlation length scale in glasses

\section{References}

[1] Gardner E 1985 Nuclear Physics B 257747

[2] Gross D J, Kanter I and Sompolinsky H 1985 Phys. Rev. Lett. 55 304-307

[3] Berthier L, Biroli G, Charbonneau P, Corwin E I, Franz S and Zamponi F 2019 The Journal of Chemical Physics 151010901

[4] Scalliet C and Berthier L 2019 Phys. Rev. Lett. 122255502

[5] Artiaco C, Baldan P and Parisi G 2020 Phys. Rev. E 101(5) 052605

[6] Seoane Beatriz and Zamponi,Francesco 2018 Soft Matter 145222

[7] Liao Q and Berthier L 2019 Phys. Rev. X 9011049

[8] Urbani P and Biroli G 2015 Phys. Rev. B 91100202

[9] Moore M A and Bray A J 2011 Phys. Rev. B 83224408

[10] Wang W, Moore M A and Katzgraber H G 2018 Phys. Rev. E 97032104

[11] Mattsson J, Jonsson T, Nordblad P, ArugaKatori H and Ito A 1995 Phys. Rev. Lett. 744305

[12] Scalliet C, Berthier L and Zamponi F 2019 Phys. Rev. E 99012107

[13] Scalliet C, Berthier L and Zamponi F 2019 Nature Communications 105102

[14] O'Hern C S, Silbert L E, Liu A J and Nagel S R 2003 Phys. Rev. E 68(1) 011306

[15] Ikeda A and Berthier L 2015 Phys. Rev. E 92012309

[16] Liu A J and Nagel S R 2010 Annual Review of Condensed Matter Physics 1 347-369

[17] Ozawa M, Berthier L and Coslovich D 2017 SciPost Phys. 3(4) 027

[18] Robinson J F, Godfrey M J and Moore M A 2016 Phys. Rev. E 93032101

[19] Godfrey M J and Moore M A 2015 Phys. Rev. E 91022120

[20] Godfrey M J and Moore M A 2014 Phys. Rev. E 89032111

[21] Bowles R K and Saika-Voivod I 2006 Phys. Rev. E 73011503

[22] Yamchi M Z, Ashwin S S and Bowles R K 2012 Phys. Rev. Lett. 109225701

[23] Ashwin S S, Zaeifi Yamchi M and Bowles R K 2013 Phys. Rev. Lett. 110145701

[24] Yamchi M Z, Ashwin S S and Bowles R K 2015 Phys. Rev. E 91022301

[25] Kofke D A and Post A J 1993 J. Chem. Phys. 984853

[26] Varga S, Balló G and Gurin P 2011 Journal of Statistical Mechanics: Theory and Experiment 2011 P11006

[27] Gurin P and Varga S 2013 J. Chem. Phys. 139244708

[28] Hu Y, Fu L and Charbonneau P 2018 Molecular Physics 1163345

[29] Hicks C L, Wheatley M J, Godfrey M J and Moore M A 2018 Phys. Rev. Lett. 120(22) 225501

[30] Huerta A, Bryk T and Trokhymchuk A 2019 arXiv e-prints arXiv:1904.05970 (Preprint 1904. 05970)

[31] Brito C and Wyart M 2006 Europhysics Letters (EPL) 76 149-155

[32] Wu Y, Karimi K, Maloney C E and Teitel S 2017 Phys. Rev. E 96032902

[33] Silbert L E, Liu A J and Nagel S R 2009 Phys. Rev. E 79021308

[34] DeGiuli E, Düring G, Lerner E and Wyart M 2014 Soft Matter 105085

[35] DeGiuli E, Laversanne-Finot A, Dring G, Lerner E and Wyart M 2014 Soft Matter 105628

[36] Ikeda A, Berthier L and Biroli G 2013 J. Chem. Phys. 138 12A507

[37] DeGiuli E, Lerner E and Wyart M 2015 The Journal of Chemical Physics 142164503 